\title{
Allowed and Forbidden d-d Transitions in Poly(3,5-dimethylpyrazolyl)methane Complexes of Nickel(II) ${ }^{\dagger}$
}

\author{
Marie-Christine Nolet, Annie Michaud, Cheryl Bain, Davit Zargarian and Christian Reber* \\ Département de Chimie, Université de Montréal, Montréal, Canada
}

Received 28 June 2005; accepted 20 September 2005; published online 22 September 2005 DOI: 10.1562/2005-06-28-RA-593

\begin{abstract}
Absorption spectra of four nickel(II) complexes with poly (pyrazolyl)methane ligands are presented in the NIR-VIS-UV region and the band system corresponding to the lowestenergy spin-allowed and spin-forbidden transitions is analyzed. A quantitative theoretical model involving coupled electronic states provides precise energies for the lowestenergy triplet and singlet excited states and allows comparisons between complexes with a variable number of nitrogen and oxygen ligator atoms. Singlet energies between 12840 and $13000 \mathrm{~cm}^{-1}$ are determined for heteroleptic complexes. These energies are in an intermediate range between those for homoleptic complexes with either nitrogen or oxygen ligator atoms with singlet states at approximately 12000 and 14000 $\mathrm{cm}^{-1}$, respectively. The new theoretical approach is compared to the traditional ligand-field parameters obtained from the maxima of the broad, spin-allowed absorption bands.
\end{abstract}

\section{INTRODUCTION}

Poly(pyrazolyl)alkane ligands and their metal complexes are used extensively in inorganic and bioinorganic chemistry because of their interesting chemical reactivity and suitability as model compounds. (1-3) Although the electronic structure of these complexes is an important factor determining these properties, only a few investigations of their electronic spectroscopy have been reported. (3-6)

Nickel(II) forms a wide variety of different complexes with poly(pyrazolyl)alkane ligands. The coordination number for these $\mathrm{d}^{8}$ complexes is six and distorted octahedral coordination geometries are observed, with the deviations from perfect octahedral structure imposed by the poly(pyrazolyl)alkane ligands, coordinated through their nitrogen ligator atoms. The number and type of poly(pyrazolyl)alkane ligands are easily varied.

We report and analyze the UV-VIS-NIR absorption spectra of four nickel(II) complexes with bis(3,5-dimethylpyrazolyl)methane

* To whom correspondence should be addressed: Département de chimie, Université de Montréal, C.P. 6128, Succ. Centre-ville, Montréal QC H3C 3J7, Canada. Fax: +1 514343 7586; e-mail: Christian.Reber@ UMontreal.ca

$\dagger$ This paper is part of a special issue dedicated to Professor J. C. (Tito) Scaiano on the occasion of his 60th birthday.

Abbreviations: bpm*, bis(3,5-dimethylpyrazolyl)methane; tpm*, tris(3,5dimethylpyrazolyl)methane.

(C) 2006 American Society for Photobiology 0031-8655/06 (bpm*) and tris(3,5-dimethylpyrazolyl)methane (tpm*) ligands. All structures are shown schematically in Chart 1 . These ligands offer an opportunity to vary the coordination sphere from three nitrogen and three oxygen atoms in $\left[(\mathrm{tpm} *) \mathrm{Ni}\left(\eta^{1}-\mathrm{NO}_{3}\right)\left(\eta^{2}-\mathrm{NO}_{3}\right)\right]$ (1) to four nitrogen and two oxygen atoms in $\left[(\mathrm{bpm} *)_{2} \mathrm{Ni}\left(\eta^{2}-\mathrm{NO}_{3}\right)\right] \mathrm{NO}_{3}$ (2), to five nitrogen and one oxygen atom in $\left[\left(\mathrm{tpm}^{*}\right)\left(\mathrm{bpm}^{*}\right) \mathrm{Ni}\left(\eta^{1}-\right.\right.$ $\left.\mathrm{NO}_{3}\right) \mathrm{NO}_{3}(3)$ and finally to six nitrogen atoms in $\left[(\mathrm{tpm} *)_{2} \mathrm{Ni}\right] \mathrm{I}_{2}$ (4). The absorption spectra are analyzed to examine to what extent trends such as the empirical spectrochemical and nephelauxetic series $(7,8)$ - both now correlated and reformulated with advanced electronic structure calculations such as density functional theory (9) — can be used to rationalize the variations observed within complexes 1 to 4 . A theoretical model recently developed by Neuhauser et al. (10) is applied and shown to be a useful tool to obtain precise excited-state energies from complete absorption band systems, an intrinsic advantage over traditional models that use only band maxima with often significant uncertainties. This new approach is shown to be successful for variations among closely related compounds, as is the case for the series of complexes 1 to 4 .

\section{MATERIALS AND METHODS}

The syntheses, characterization and crystal structures of complexes $\mathbf{1}$ to $\mathbf{3}$ (A. Michaud, F.-G. Fontaine, and D. Zargarian, in press, Inorg. Chim . Acta [2006], doi:10.1016/j.ica.2005.09.046) and 4 (11) are described in the literature. Absorption spectra of complexes $\mathbf{1}$ to $\mathbf{4}$ in acetonitrile solution were measured with a Cary $5 \mathrm{E}$ spectrometer (Varian Inc., Palo Alto, CA) with the spectral resolution set to $2 \mathrm{~nm}$ from $1600 \mathrm{~nm}$ to $900 \mathrm{~nm}$ and to 1 $\mathrm{nm}$ for wavelengths shorter than $900 \mathrm{~nm}$. Raman spectra of solid samples were measured with a Raman microscope (Renishaw System 3000, Wottonunder-Edge, Gloucestershire, UK) using the 488.0 and $514.5 \mathrm{~nm}$ Argon ion laser lines as excitation sources. The resolution of the Raman spectra is 1 $\mathrm{cm}^{-1}$. The instrumental resolution of both spectrometers is higher by an order of magnitude than the narrowest features observed in the spectra.

\section{SPECTROSCOPIC RESULTS}

Solution absorption spectra of the nickel(II) complexes $\mathbf{1}$ to $\mathbf{4}$ illustrated in Chart 1 are shown in Fig. 1. The low molar absorptivities of 5 to $15 \mathrm{M}^{-1} \mathrm{~cm}^{-1}$ measured for the broad bands in these spectra are typical for the three spin-allowed $d-d$ transitions in sixcoordinate, exactly or approximately octahedral complexes of nickel(II), as documented in a detailed compilation. (12) The band maxima for complexes $\mathbf{1}$ to $\mathbf{4}$ are summarized in Table 1 and compared to those for selected reference compounds. The bands are easily assigned from the Tanabe-Sugano diagram for octahedral complexes with the $\mathrm{d}^{8}$ electron configuration, shown in Fig. 2. The ground state is ${ }^{3} \mathrm{~A}_{2 \mathrm{~g}}$ and three spin-allowed transitions to the ${ }^{3} \mathrm{~T}_{2 \mathrm{~g}}$, 


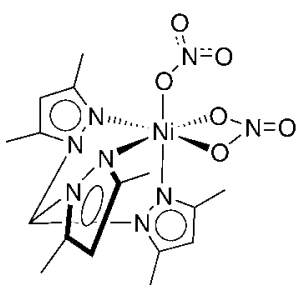

1

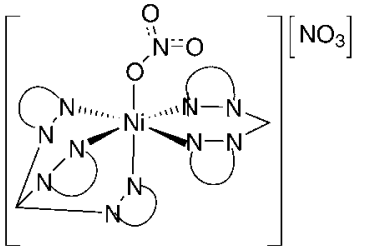

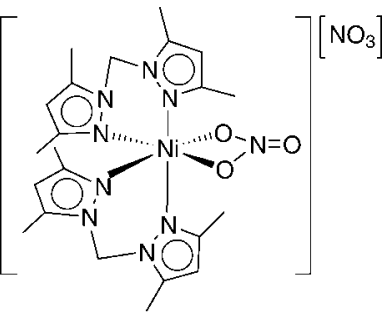

2

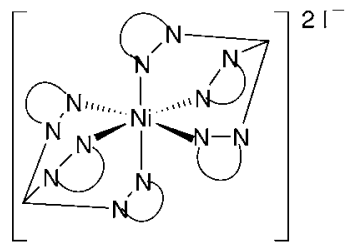

4
Chart 1. Schematic structures of complexes 1 to 4 .

${ }^{3} \mathrm{~T}_{1 \mathrm{~g}}\left({ }^{3} \mathrm{~F}\right)$ and ${ }^{3} \mathrm{~T}_{1 \mathrm{~g}}\left({ }^{3} \mathrm{P}\right)$ excited states are observed. Broad bands are expected for all these transitions, because the excited states arise from electron configurations different from the ground state configuration. Symmetry labels for an idealized octahedral structure are used throughout this analysis. Point-group symmetries of the nickel(II) sites determined by single-crystal X-ray diffraction are $\mathrm{C}_{1}$ for complexes 1 and 2 (A. Michaud, F.-G. Fontaine, and D. Zargarian, accepted for publication), $\mathrm{C}_{\mathrm{s}}$ for complex 3 (A. Michaud, F.-G. Fontaine, and D. Zargarian, accepted for publication) and $\mathrm{C}_{2 \mathrm{~h}}$ for complex 4 (11). Deviations from octahedral symmetry caused by of the mixed ligand spheres for complexes $\mathbf{1}$ to 3 do not lead to multiple band maxima for any of the spinallowed bands in Fig. 1. A formal reason for this is the high holohedrized symmetry of the ligand-field potential (8) in these complexes. The holohedrized symmetry, obtained for orthoaxial complexes by replacing individual ligands along one axis by their average ligand-field strength, is $\mathrm{O}_{h}$ for complex 1 and $\mathrm{D}_{4 h}$ for complexes $\mathbf{2}$ and $\mathbf{3}$, with very similar total ligand field strengths along the three axes, indicating that any splitting of the ${ }^{3} \mathrm{~T}_{2 \mathrm{~g}}$ and ${ }^{3} \mathrm{~T}_{1 \mathrm{~g}}$ states for octahedral symmetry should be small. All holohedrized symmetries contain a center of inversion, rationalizing the low molar absorptivities as a consequence of the parity selection rule. Complex $\mathbf{4}$ also has $\mathrm{O}_{\mathrm{h}}$ holohedrized symmetry, but shows a particularly broad ${ }^{3} \mathrm{~T}_{1 \mathrm{~g}}\left({ }^{3} \mathrm{~F}\right)$ absorption band with a width at half height of $3340 \mathrm{~cm}^{-1}$, significantly larger than the corresponding bands in $\left.[\mathrm{Ni} \text { (ethylenediamine })_{3}\right]^{2+}$ and $[\mathrm{Ni}(o$-phenanthroline $\left.)_{3}\right]^{2+}$, complexes with strong trigonal distortions where widths of 1880 and $2030 \mathrm{~cm}^{-1}$ are observed. $(13,14)$ Both this large width and the significantly narrower ${ }^{3} \mathrm{~T}_{2 \mathrm{~g}}$ band have been rationalized with angular overlap calculations based on singlecrystal spectra measured at low temperature (5), which show multiple resolved maxima for this band, as a consequence of the strongly anisotropic nickel(II)-pyrazolyl $\pi$ bonding in $\left.[\mathrm{Ni} \text { (tris(pyrazolyl)methane) })_{2}\right]^{2+}$, a complex closely related to 4 .

A single band corresponding to a spin-forbidden transition is observed at approximately $13000 \mathrm{~cm}^{-1}$ on the high-energy side of the lowest-energy spin-allowed band for all complexes. Transitions to other singlet states are too weak to be observed in the spectra in Fig. 1. Inspection of the Tanabe-Sugano diagram in Fig. 2 indicates

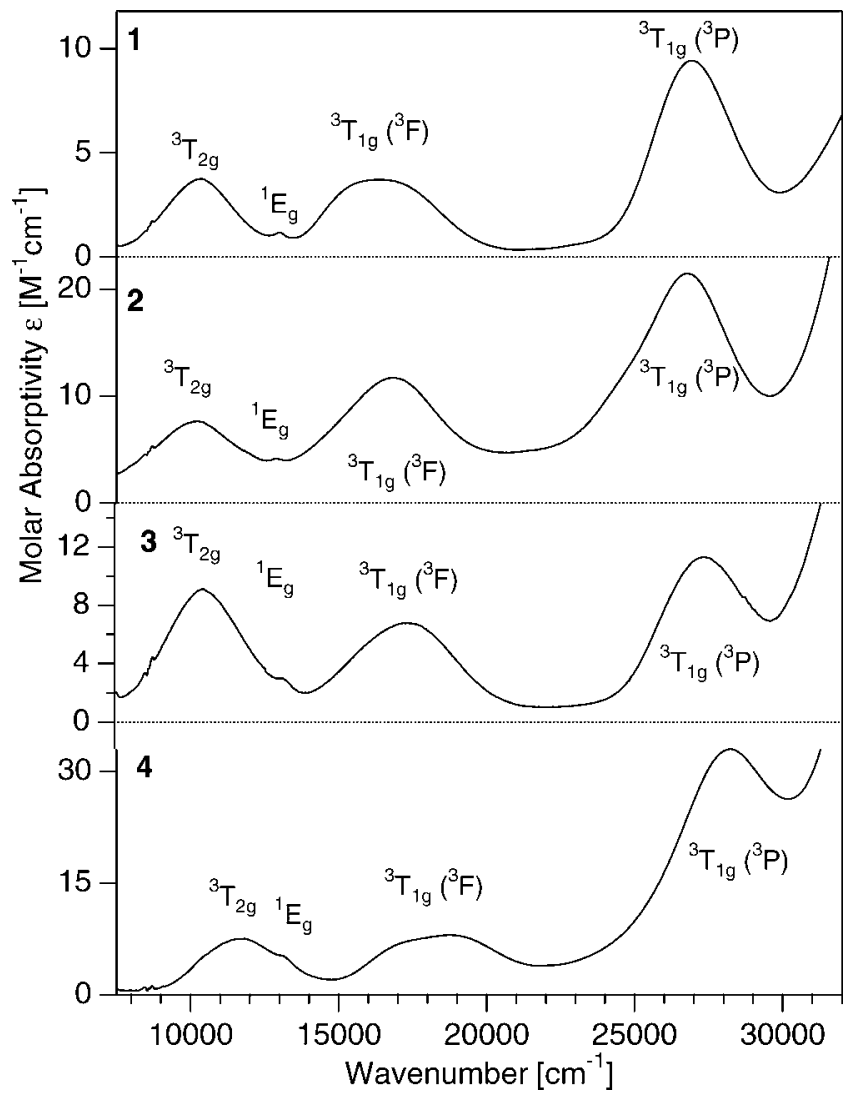

Figure 1. Absorption spectra in acetonitrile solution of $\left[(\operatorname{tpm} *) \mathrm{Ni}\left(\mathrm{NO}_{3}\right)_{2}\right]$ (1), $\left[(\mathrm{bpm} *)_{2} \mathrm{Ni}\left(\mathrm{NO}_{3}\right)\right]^{+}(\mathbf{2}),\left[(\mathrm{tpm} *)(\mathrm{bpm} *) \mathrm{Ni}\left(\mathrm{NO}_{3}\right)\right]^{+}(\mathbf{3})$, and $\left[\left(\mathrm{tpm}^{*}\right)_{2} \mathrm{Ni}\right]^{2+}(\mathbf{4})$

that the final state of this transition is ${ }^{1} \mathrm{E}_{\mathrm{g}}$, the lowest-energy singlet state. It arises from the same electron configuration as the ground state and therefore a narrow band is expected.

The band maxima of the spin-allowed transitions are traditionally used to calculate the ligand field parameters $10 \mathrm{Dq}$ and $\mathrm{B}$ with the following equations: $(13,15)$

$$
\begin{aligned}
10 \mathrm{Dq} & =\mathrm{E}\left({ }^{3} \mathrm{~A}_{2 \mathrm{~g}} \rightarrow{ }^{3} \mathrm{~T}_{2 \mathrm{~g}}\right) \\
15 \mathrm{~B} & =\mathrm{E}\left({ }^{3} \mathrm{~A}_{2 \mathrm{~g}} \rightarrow{ }^{3} \mathrm{~T}_{1 \mathrm{~g}}\left({ }^{3} \mathrm{~F}\right)\right)+\mathrm{E}\left({ }^{3} \mathrm{~A}_{2 \mathrm{~g}} \rightarrow{ }^{3} \mathrm{~T}_{1 \mathrm{~g}}\left({ }^{3} \mathrm{P}\right)\right)-30 \mathrm{Dq}
\end{aligned}
$$

All calculated values for $10 \mathrm{Dq}$ and $\mathrm{B}$ are summarized in Table 1 . The $10 \mathrm{Dq} / \mathrm{B}$ ratios of $11-15$ for $\mathbf{1}$ to $\mathbf{4}$ are higher than for homoleptic complexes with oxygen ligator atoms, illustrated by $\left[\mathrm{Ni}\left(\mathrm{H}_{2} \mathrm{O}\right)_{6}\right]^{2+}$ at a $10 \mathrm{Dq} / \mathrm{B}$ ratio of $9.2(16)$ in Fig. 2, and lower than the ratio of 18 for complexes with strong-field ligands such as $\left[\mathrm{Ni}(o \text {-phenanthroline })_{3}\right]^{2+}(13-15)$, also included on the abscissa of Fig. 2. It has been pointed out for several complexes $(4,5,17)$ that tris(pyrazolyl) ligands appear to be somewhat lower in the spectrochemical series than ligands such as bipyridine or phenanthroline, but higher than monodentate pyrazole ligands. (18) The maxima of the lowest-energy spin-allowed band for nickel(II) complexes with tris(pyrazolyl)borate ligands (19) have been reported recently. (20) A value of $11400 \mathrm{~cm}^{-1}$ was obtained for [bis(tris(3,5dimethylpyrazolyl)borate)nickel(II)], lower by only $270 \mathrm{~cm}^{-1}$ than for complex 4. In contrast, the homologue [bis(tris(pyrazolyl)borate)nickel(II)] with unsubstituted pyrazolyl groups has a band maximum at $11900 \mathrm{~cm}^{-1}$, higher by $230 \mathrm{~cm}^{-1}$ than complex 4 . This comparison shows that the energies of spin-allowed 
Table 1. Spin-allowed band maxima measured at room temperature in solution and crystal-field parameter values for 10Dq and B calculated from Eqs. 1 and 2 for complexes 1 to 4 . All values are in $\mathrm{cm}^{-1}$ except the $10 \mathrm{Dq} / \mathrm{B}$ ratios, which are dimensionless

\begin{tabular}{|c|c|c|c|c|c|c|}
\hline Complex & ${ }^{3} \mathrm{~A}_{2 \mathrm{~g}} \rightarrow{ }^{3} \mathrm{~T}_{2 \mathrm{~g}}$ & ${ }^{3} \mathrm{~A}_{2 \mathrm{~g}} \rightarrow{ }^{3} \mathrm{~T}_{1 \mathrm{~g}}\left({ }^{3} \mathrm{~F}\right)$ & ${ }^{3} \mathrm{~A}_{2 \mathrm{~g}} \rightarrow{ }^{3} \mathrm{~T}_{1 \mathrm{~g}}\left({ }^{3} \mathrm{P}\right)$ & 10Dq & $\mathrm{B} \dagger$ & 10Dq/B \\
\hline$\left[\left(\mathrm{tpm}^{*}\right) \mathrm{Ni}\left(\mathrm{NO}_{3}\right)_{2}\right](\mathbf{1})$ & 10300 & 16410 & 27040 & 10300 & 837 & 12.3 \\
\hline$\left[\left(\mathrm{bpm}^{*}\right)_{2} \mathrm{Ni}\left(\mathrm{NO}_{3}\right)\right]^{+}(\mathbf{2})$ & 10170 & 16800 & 26750 & 10170 & 869 & 11.7 \\
\hline$\left[\left(\mathrm{bpm}^{*}\right)(\mathrm{tpm} *) \mathrm{Ni}\left(\mathrm{NO}_{3}\right)\right]^{+}(\mathbf{3})$ & 10420 & 17264 & 27366 & 10420 & 891 & 11.7 \\
\hline$\left[(\operatorname{tpm} *)_{2} \mathrm{Ni}\right]^{2+}(\mathbf{4})$ & 11670 & 18470 & 28330 & 11670 & 786 & 14.8 \\
\hline$\left[\mathrm{Ni}(\text { pyrazole })_{6}\right]^{2+}+$ & 10650 & 17100 & 27500 & 10650 & 843 & 12.6 \\
\hline$\left[\mathrm{Ni}\left(\mathrm{NH}_{3}\right)\right]_{6}^{2+} \S$ & 10730 & 17530 & 28110 & 10730 & 830 & 12.9 \\
\hline$\left[\mathrm{Ni}(o \text {-phenanthroline })_{3}\right]^{2+} \|$ & 12690 & 19050 & n/a & 12690 & 710 & 17.9 \\
\hline$\left[\mathrm{Ni}\left(\mathrm{H}_{2} \mathrm{O}\right)_{6}\right]^{2+} \S$ & 8580 & 14300 & 25370 & 8580 & 929 & 9.2 \\
\hline
\end{tabular}

$\dagger \mathrm{B}$ is $1082 \mathrm{~cm}^{-1}$ for the free $\mathrm{Ni}(\mathrm{II})$ ion.

$\$(18)$.

$\S(16)$.

|| (14).

transitions for poly(pyrazolyl)methane and poly(pyrazolyl)borate ligands are very similar and vary by amounts on the same order of magnitude as those for different alkyl substituents on the pyrazolyl groups. The 10Dq/B ratios for complexes $\mathbf{1}$ to $\mathbf{3}$ are between 11.7 and 12.3 , very similar to the ratio of 12.2 reported for [ $(\mathrm{bis}(3,5-$ dimethylpyrazol-1-ylomethyl)aminoethane) $\left.\mathrm{Ni}\left(\eta^{1}-\mathrm{NO}_{3}\right)\left(\eta^{2}-\mathrm{NO}_{3}\right)\right]$, (17) as expected from the combination of nitrogen and oxygen ligator atoms, and also to $\left[\mathrm{Ni}\left(\mathrm{NH}_{3}\right)_{6}\right]^{2+}$ and $\left[\mathrm{Ni}(\text { pyrazole })_{6}\right]^{2+}$, for which ratios of 12.9 and 12.2 have been determined. $(13,16,18)$ These variations indicate that the $10 \mathrm{Dq} / \mathrm{B}$ ratio is not a very sensitive quantity for classifying complexes 1 to $\mathbf{4}$, because similar values are obtained for chemically very different ligands.

The Raman spectra of complexes $\mathbf{1}$ to $\mathbf{4}$ show multiple distinct peaks in the region of the metal-ligand stretching modes. All vibrational energies between 200 and $600 \mathrm{~cm}^{-1}$ are listed in Table 2, but unambiguous assignments to either metal-ligand stretching modes or low-frequency ligand-centered vibrational modes can not be made. In contrast, the Raman-active $v_{3}(E)$ vibrational mode of the nitrate anion can be used to confirm the coordination of the $\mathrm{NO}_{3}^{-}$ligands in complexes $\mathbf{1}$ to $\mathbf{3}$. Complex $\mathbf{4}$ does not contain nitrate and can be used to assign Raman peaks to modes of the poly(pyrazolyl)methane ligands. The $v_{3}(\mathrm{E})$ frequency of $\mathrm{NO}_{3}^{-}$is $1390 \mathrm{~cm}^{-1}$ in the uncoordinated anion (21), and a peak is observed at $1395 \mathrm{~cm}^{-1}$ in complexes $\mathbf{2}$ and $\mathbf{3}$, assigned to the $\mathrm{NO}_{3}^{-}$counterions. The degeneracy of this mode is lifted for coordinated nitrate ligands and two bands are observed. In compounds $\mathbf{1}$ to $\mathbf{3}$ they are separated by approximately $150 \mathrm{~cm}^{-1}$, as summarized in Table 2. The frequencies for both monodentate and bidentate coordination are within the characteristic ranges established from Raman (22) and IR (23) spectroscopy, and in agreement with the monodentate and bidentate coordination for the nitrate ligands defined by the crystal structures and shown in Chart 1 for complexes 1 to 3 .

\section{DISCUSSION}

\section{Traditional ligand-field parameters}

Complexes 1 to $\mathbf{4}$ show spin-allowed band maxima higher in energy than those for homoleptic complexes with oxygen ligator atoms and lower in energy than those observed for complexes with nitrogen ligator atoms. $(13,14,23)$ The values of $10 \mathrm{Dq} / \mathrm{B}$ in Table 1 illustrate this trend, in particular when compared to the homoleptic complexes included in the Table and on the abscissa of Fig. 2.
Within the series of compounds $\mathbf{1}$ to $\mathbf{4}$, it is not obvious to establish the expected systematic trends along the spectrochemical and nephelauxetic series (7-9) from the values of 10Dq and B in Table 1. Compound 4 has the highest value of $10 \mathrm{Dq}, 11670 \mathrm{~cm}^{-1}$, as expected for its six nitrogen ligator atoms. According to the

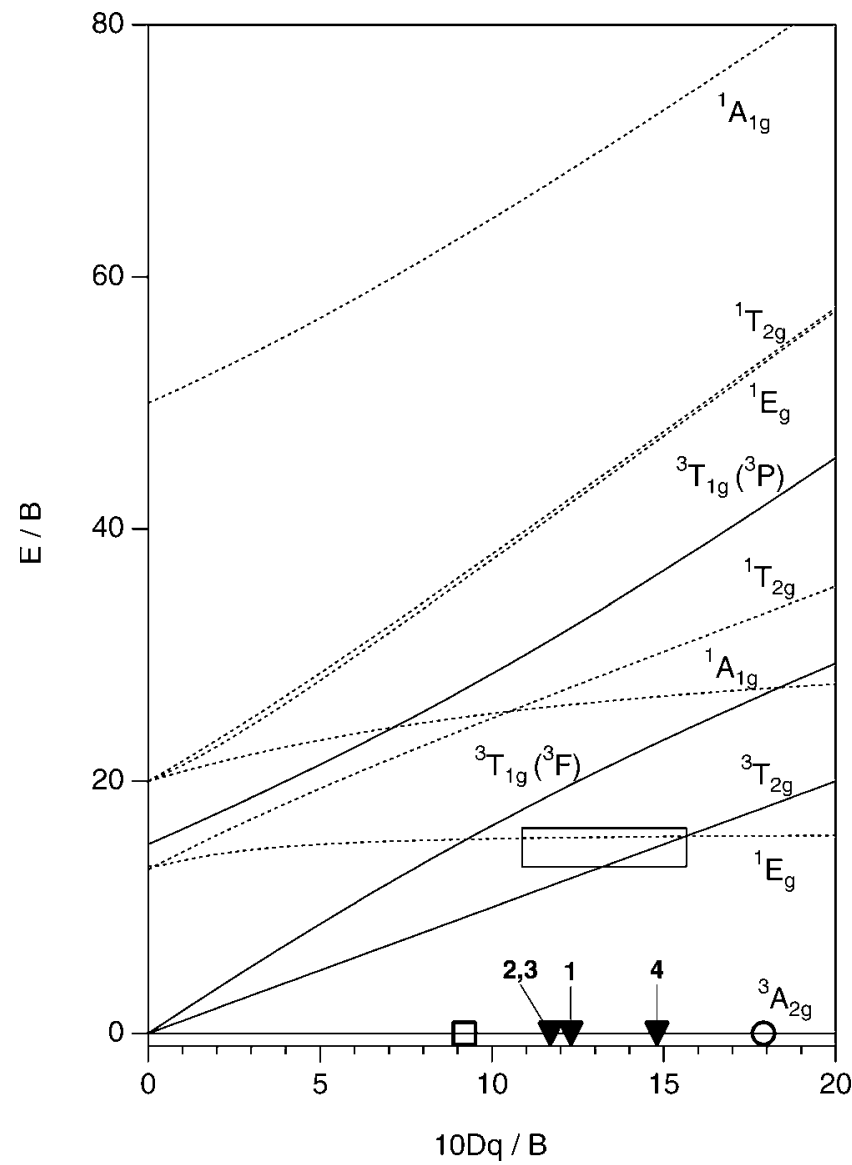

Figure 2. Tanabe-Sugano diagram for the $d^{8}$ configuration. Triplet and singlet states are given as solid and dotted curves, respectively. The positions of complexes $\mathbf{1}$ to $\mathbf{4}$ on the 10Dq/B abscissa are denoted by solid triangles, based on the values in Table 1. Literature values for $\left[\mathrm{Ni}\left(\mathrm{H}_{2} \mathrm{O}\right)_{6}\right]^{2+}$ (open square) and $\left[\mathrm{Ni}(o \text {-phenanthroline })_{3}\right]^{2+}$ (open circle) are given for comparison. The rectangle indicates the region of close energetic proximity for the lowest triplet and singlet excited states examined for complexes 1 to 4 . 
Table 2. Selected Raman frequencies in $\mathrm{cm}^{-1}$

\begin{tabular}{|c|c|c|c|c|}
\hline Compound & Ni-ligand stretching region & $\mathrm{NO}_{3}^{-}, v_{3}(\mathrm{E})$ counterion $\dagger$ & $\mathrm{NO}_{3}^{-}, v_{3}$ monodentate $\ddagger$ & $\mathrm{NO}_{3}{ }^{-}, v_{3}$ chelate $\S$ \\
\hline$\left[(\mathrm{tpm} *) \mathrm{Ni}\left(\mathrm{NO}_{3}\right)_{2}\right](\mathbf{1})$ & $222,258,327,349,406,423,442,577$ & $\mathrm{n} / \mathrm{a}$ & 1309,1460 & 1325,1482 \\
\hline$\left[\left(\mathrm{bpm}^{*}\right)_{2} \mathrm{Ni}\left(\mathrm{NO}_{3}\right)\right]^{+}(\mathbf{2})$ & $229,250,285,367,435$ & 1395 & $\mathrm{n} / \mathrm{a}$ & 1305,1480 \\
\hline$\left[\left(\mathrm{bpm}^{*}\right)(\mathrm{tpm} *) \mathrm{Ni}\left(\mathrm{NO}_{3}\right)\right]^{+}(\mathbf{3})$ & $216,347,383,416,480,491$ & 1396 & 1275,1459 & $\mathrm{n} / \mathrm{a}$ \\
\hline$\left[(\mathrm{tpm} *)_{2} \mathrm{Ni}\right]^{2+}(\mathbf{4})$ & $225,242,342,383,404,423,488$ & $\mathrm{n} / \mathrm{a}$ & $\mathrm{n} / \mathrm{a}$ & $\mathrm{n} / \mathrm{a}$ \\
\hline
\end{tabular}

$+1390 \mathrm{~cm}^{-1}(21)$.

$\$ 1253-1290 \mathrm{~cm}^{-1}, 1481-1531 \mathrm{~cm}^{-1}(22)$.

$\S 1230-1350 \mathrm{~cm}^{-1}, 1480-1650 \mathrm{~cm}^{-1}(22)$.

spectrochemical series, values for $10 \mathrm{Dq}$ should decrease if nitrogen ligator atoms are replaced by oxygen atoms. Compound $\mathbf{1}$, with the largest number of oxygen ligator atoms among the four complexes studied here, has a 10Dq value of $10300 \mathrm{~cm}^{-1}$, significantly lower than 4 . In contrast, the intermediate complexes $\mathbf{2}$ and $\mathbf{3}$, with two and one oxygen ligator atoms, respectively, do not show a continuous increase of $10 \mathrm{Dq}$.

These irregular trends can be rationalized qualitatively by considering nickel(II)-N(pyrazolyl) bond lengths in tpm*, bpm* and pyrazole complexes. The longest bonds are observed for the $\left[\mathrm{Ni}(\text { pyrazole })_{6}\right]^{2+}$ complex, where average values of $2.12 \AA$ have been reported (18). Average bond lengths for [bis(tris(pyrazolyl) methane)Ni] are on the order of 2.07-2.08 $\AA$ and appear to be independent of the number of methyl substituents on the pyrazolyl groups. $(5,11)$ Values of the ligand field parameter 10Dq for these complexes are given in Table 1 and reflect this difference: 10Dq increases by approximately $10 \%$ from $\left[\mathrm{Ni}(\text { pyrazole })_{6}\right]^{2+}$ to complex 4. The corresponding average nickel(II)-N(bpm*) bond lengths are slightly longer, on the order of 2.07 to $2.09 \AA$ for complex 3 and for [bis(bis(pyrazolyl)(2-thienyl)methane)(nitrato$\left.\mathrm{O}, \mathrm{O}^{\prime}\right)$ nickel(II) $]^{+}$, a complex similar to $\mathbf{3}$ in which two pyrazolyl groups of each poly(pyrazolyl) ligand are coordinated to the metal center. (6) These slightly longer bonds are a likely reason for the lower value of 10Dq for complex $\mathbf{2}$ compared to complex $\mathbf{1}$ and put $\left(\mathrm{bpm}^{*}\right)$ at an intermediate ligand field strength between pyrazole and (tpm*).

The values of $\mathrm{B}$ for compounds $\mathbf{1}$ and $\mathbf{4}$ show the decrease expected from the nephelauxetic series for the change from a coordination sphere formed by three oxygen and three nitrogen ligator atoms to a homoleptic coordination sphere formed by six nitrogen atoms. Again, compounds $\mathbf{2}$ and $\mathbf{3}$ do not follow this tendency, showing an increase of B with increasing number of nitrogen ligator atoms, in marked contrast to the nephelauxetic series and casting doubt on the reliability of trends for the values of interelectronic repulsion parameters for similar complexes determined from the broad absorption maxima in solution spectra. The variations for both $10 \mathrm{Dq}$ and $\mathrm{B}$ show the limitations of traditional ligand-field procedures for the characterization of relatively small variations in the coordination sphere, as is the case in the series of compounds studied here.

One obvious improvement of the ligand-field model, attempted for several poly(pyrazolyl) complexes in the literature, is to use the exact angular positions of the ligator atoms with the angular overlap model. (3-6) This approach leads to a large number of additional parameters that cannot be derived from the limited number of transitions observed in the spectra in Fig. 1, in particular for the complexes with a mixed ligand sphere. In view of the large number of angular-overlap parameters, the Racah parameter B was usually held constant for different complexes analyzed with this model, a procedure not acceptable for our goal of characterizing the variation of both $10 \mathrm{Dq}$ and the electron-electron interaction parameter B. Another omission in the determination of $10 \mathrm{Dq}$ and B with Eqs. 1 and 2 is spin-orbit coupling, traditionally not considered to have a significant influence on the absorption band maxima. In the following, we present an alternative approach to characterizing the ligand field strength and electron-electron interaction based on the lowest-energy singlet and triplet excited states, including their significant interaction through spin-orbit coupling, which is quantitatively included in the model.

\section{Coupled singlet and triplet excited states}

A prominent feature of the absorption spectra in Fig. 1 is the shoulder near the lowest-energy spin-allowed ${ }^{3} \mathrm{~A}_{2 \mathrm{~g}} \rightarrow{ }^{3} \mathrm{~T}_{2 \mathrm{~g}}$ band, assigned as the spin-forbidden ${ }^{3} \mathrm{~A}_{2 \mathrm{~g}} \rightarrow{ }^{1} \mathrm{E}_{\mathrm{g}}$ transition. The close proximity of these two excited states leads to strong mixing through spin-orbit coupling, an effect that has been analyzed in detail for a number of homoleptic complexes of nickel(II). $(13,14,16,24,25)$ The mixing causes an increase of the intensity and bandwidth of the spin-forbidden transition, leading to a band that is easy to detect in the spectra in Fig. 1, in contrast to all other transitions to singlet excited states, which are not observed in Fig. 1. An important consequence of the coupled excited states is the change of band shapes, which also influences the energies of the band maxima. In order to analyze these effects, a quantitative model has to be used, and calculated spectra have to be fitted to experimental data. We apply a theoretical model to calculate the absorption spectrum of a forbidden spin-flip transition close in energy to an allowed interconfigurational band. This model successfully reproduces the spectra of many high-symmetry homoleptic complexes of nickel(II) and chromium(III) where coupled electronic states of different multiplicity occur. $(10,14,25)$ In the following, we apply it for the first time to lower-symmetry, heteroleptic complexes. Figure 3 illustrates the model and all parameters used for the analysis.

The potential energy curves for the ${ }^{3} \mathrm{~A}_{2 \mathrm{~g}}$ ground state as well as the ${ }^{1} \mathrm{E}_{\mathrm{g}}$ and ${ }^{3} \mathrm{~T}_{2 \mathrm{~g}}$ excited states are given by the solid curves in Fig. 3 , calculated from ground state vibrational frequencies $\omega_{0}$ obtained experimentally from the Raman spectra summarized in Table 2 and using the harmonic approximation. Identical frequencies were used for all electronic states in Fig. 3. We use the symbols for all relevant quantities defined in the original publication of this model (10). The energy minimum of the ground-state curve corresponds to the equilibrium geometry. Because no metal-ligand bonding changes occur in the singlet state, its minimum is placed at the same position as the ground state along the normal coordinate in 
Fig. 3. The minimum for the triplet excited state is offset by $x_{\mathrm{A}}$. The Franck-Condon maximum for the triplet band is given by the parameter $\Delta$, and the energy differences between the ground and excited state potential energy minima are $\varepsilon_{\mathrm{F}}$ and $\varepsilon_{\mathrm{A}}$ for the singlet and triplet excited states, respectively. The two excited states are coupled by the constant $\gamma$, expected to be similar in magnitude to the spin-orbit coupling constant for first-row transition metal ions and leading to the adiabatic potential energy surfaces shown as dotted lines in Fig. 3, different from the harmonic diabatic curves. Both sets of curves are necessary to calculate the absorption band system arising from transitions to these coupled excited states. The Hamiltonian for the two excited states described by the coupled potential energy surfaces is given by:

$$
\mathrm{H}=\frac{\mathrm{p}^{2}}{2 \mathrm{M}}\left[\begin{array}{ll}
1 & 0 \\
0 & 1
\end{array}\right]+\left[\begin{array}{cc}
\frac{1}{2} \mathrm{M} \omega_{0}^{2} x^{2}+\varepsilon_{\mathrm{F}} & \gamma \\
\gamma & \frac{1}{2} \mathrm{M} \omega_{0}^{2}\left(x-x_{\mathrm{A}}\right)^{2}+\varepsilon_{\mathrm{A}}
\end{array}\right]
$$

Analytical and numerical solutions for the absorption spectrum resulting from transitions to these two coupled excited states have been published and discussed $(10,25)$. The calculated spectrum involving the coupled states is given by

$$
\begin{aligned}
\sigma(\omega) & =\frac{1}{2 \pi} \int_{-\infty}^{\infty}\left\langle\psi_{0}\left|\mathrm{e}^{-\mathrm{i}(\mathrm{H}-\omega) \mathrm{t}-\Gamma|\mathrm{t}|}\right| \psi_{0}\right\rangle \mathrm{dt} \\
& =\frac{1}{\pi} \operatorname{Im}\left\langle\psi_{0}\left|\mathrm{H}-\omega-\mathrm{i} \Gamma^{-1}\right| \psi_{0}\right\rangle
\end{aligned}
$$

The absorption spectra in Fig. 1 do not allow an experimental determination of all triplet excited levels arising from ${ }^{3} \mathrm{~T}_{2} \mathrm{~g}$ split by deviations from octahedral symmetry and spin-orbit coupling. We describe the bandshape for the allowed transition to the triplet excited state in the absence of coupling to the singlet excited state by a Lorentz profile with a width of $\sqrt{\omega_{0} \lambda}$. (10) With this simplification, which also makes it unnecessary to specify individual normal coordinates and offsets $\mathrm{x}_{\mathrm{A}}$, an analytical equation for the absorption spectrum is obtained:

$$
\sigma(\omega)=-\frac{1}{\pi} \operatorname{Im}\left(\frac{\beta}{1-\gamma^{2} \alpha \beta}\right)
$$

where $\alpha$ and $\beta$ are defined as:

$$
\begin{aligned}
& \alpha=\frac{1}{\omega-\varepsilon_{\mathrm{F}}+i \Gamma} \\
& \beta=\frac{1}{\omega-\Delta+\mathrm{i} \sqrt{\omega_{0} \lambda}}
\end{aligned}
$$

Spectra calculated with equation (5) are shown in the inset to Fig. 3. The spectra calculated with a value of zero for the coupling constant $\gamma$ are shown as Lorentz profiles denoted by dotted traces for two different values of $\varepsilon_{\mathrm{A}}$. The traces therefore differ only in the position of the band maximum along the wavenumber axis. The corresponding spectra calculated with a nonzero coupling constant are shown as solid traces in the inset, indicating the important influence of the energy difference between $\varepsilon_{\mathrm{A}}$ and $\varepsilon_{\mathrm{F}}$. For a small difference, a characteristic band shape with two maxima separated by a minimum, denoted as the interference dip $(10,25,26)$, is

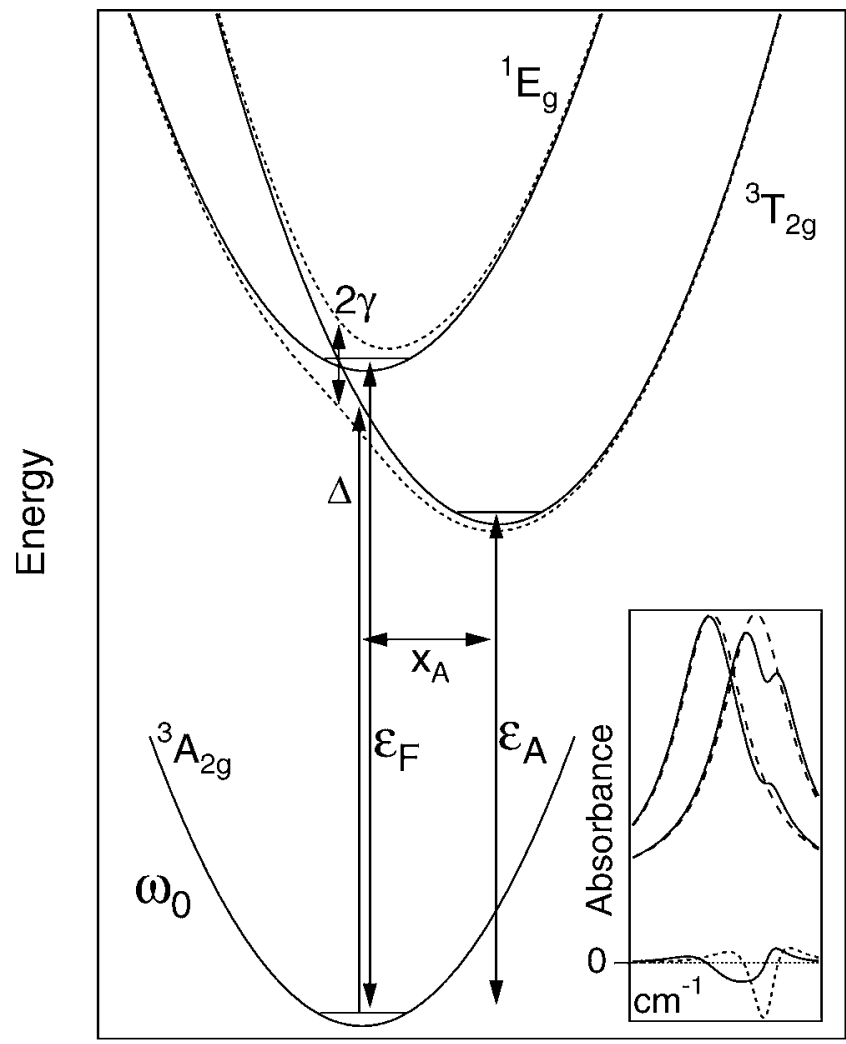

\section{Normal Coordinate}

Figure 3. Coupled potential energy surfaces for the ground state and the lowest-energy singlet and triplet excited states with definition of model parameters. The inset shows calculated spectra for two different values of $\varepsilon_{\mathrm{A}}$. Dotted and solid traces denote calculated spectra corresponding to uncoupled and coupled excited states, respectively. The difference traces at the bottom of the inset illustrate the effect of coupled excited states on the shape of the absorption spectrum. The absorption baseline is given as a thin dotted line in order to emphasize minima and maxima of the difference traces.

calculated, as illustrated by the spectrum peaking at higher energy. The difference between this spectrum and the Lorentz profile is given as a dotted trace at zero absorbance, and it shows the maximum-minimum-maximum characteristic typical for coupled excited states in molecular spectra (10), in marked contrast to atomic spectra. (27) The band shape is less obvious for the larger separation between $\varepsilon_{\mathrm{A}}$ and $\varepsilon_{\mathrm{F}}$, where a spectrum with a maximum and a shoulder is observed, also given in the inset of Fig. 2. The difference trace for this situation is given as a solid line starting at zero absorbance, and it again clearly shows the maximumminimum-maximum profile, but with a weaker low-energy maximum and a broader minimum denoting the interference dip than for the dotted difference trace. This comparison shows quantitatively that absorption spectra involving coupled excited states retain a characteristic band shape with an interference dip, even if no clearcut minimum can be distinguished in the experimental spectrum. A model using the full set of coupled potential energy curves shown in Fig. 3 has to be applied for a precise analysis of these spectra, as described in the following for complexes $\mathbf{1}$ to 4 .

In order to analyze experimental spectra with this model, as many parameters as possible are set to experimental quantities. Vibrational frequencies $\omega_{0}$ were held constant at values observed 


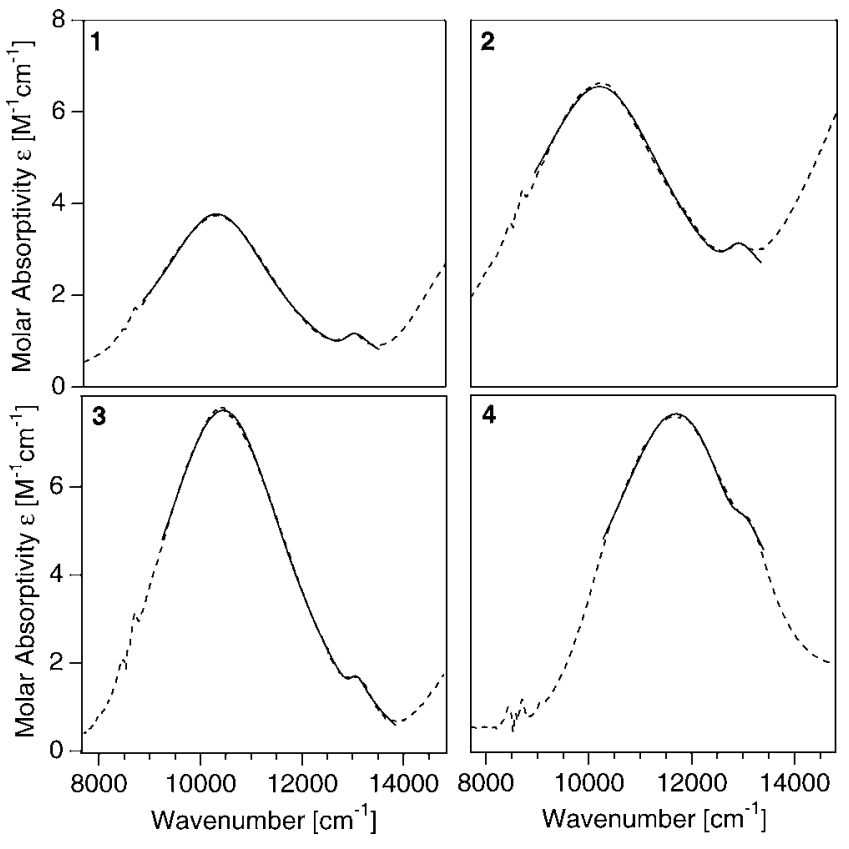

Figure 4. Calculated absorption spectra (solid lines) compared to experimental traces (dotted lines) in the region of the lowest-energy singlet and triplet transitions for complexes 1 to $\mathbf{4}$.

in the Raman spectra and initial values for $\Delta$ and $\varepsilon_{\mathrm{F}}$ were estimated from the spectra. The coupling constant $\gamma$ was limited to values below $1000 \mathrm{~cm}^{-1}$, corresponding to the order of magnitude of the spin-orbit coupling constant for nickel(II). Only $\lambda$, a factor contributing to the width of the spin-allowed absorption band, and $\Gamma$, a phenomenological parameter defining the shape of the spectrum in the region of the formally spin-forbidden band, were treated as adjustable parameters without numerical constraints. The most important parameter values obtained from the fits are band maxima $\Delta$ for the allowed transition and energies $\varepsilon_{F}$ for the lowest-energy singlet excited state of complexes 1 to $\mathbf{4}$.

Figure 4 shows a comparison of experimental and calculated spectra. The agreement is excellent, and the numerical values of all parameters used for the calculations are summarized in Table 3. We carried out calculations with several different experimental vibrational frequencies, because multiple totally-symmetric metalligand stretching modes are expected for complexes with a mixed ligand sphere, such as complexes $\mathbf{1}$ to $\mathbf{3}$, or for complexes with lower than $\mathrm{O}_{\mathrm{h}}$ point group symmetry, such as complex 4. Numerical results for two different frequencies $\omega_{0}$ from Table 2 are given in Table 3 for compounds $\mathbf{1}$ and $\mathbf{4}$. The calculated spectra

Table 3. Parameter values from fits of Eq. 5 to the absorption spectra in Fig. 4. All values are in $\mathrm{cm}^{-1}$ units. Values obtained using two different frequencies $\omega_{0}$ are given for compounds $\mathbf{1}$ and $\mathbf{4}$

\begin{tabular}{lrrrrrr}
\hline \multicolumn{1}{c}{ Compound } & \multicolumn{1}{c}{$\omega_{0}$} & \multicolumn{1}{c}{$\lambda$} & $\Gamma$ & $\gamma$ & $\varepsilon_{\mathrm{F}}$ & $\Delta$ \\
\hline$\left[(\mathrm{tpm} *) \mathrm{Ni}\left(\mathrm{NO}_{3}\right)_{2}\right](\mathbf{1})$ & 577 & 3760 & 274 & 436 & 12873 & 10389 \\
{$\left[(\mathrm{tpm} *) \mathrm{Ni}\left(\mathrm{NO}_{3}\right)_{2}\right](\mathbf{1})$} & 222 & 9760 & 274 & 397 & 12854 & 10370 \\
{$\left[\left(\mathrm{bpm}^{*}\right)_{2} \mathrm{Ni}\left(\mathrm{NO}_{3}\right)\right]^{+}(\mathbf{2})$} & 285 & 11728 & 500 & 500 & 12838 & 10305 \\
$\left.\left[\left(\mathrm{bpm}^{*}\right)(\mathrm{tpm})^{*}\right) \mathrm{Ni}\left(\mathrm{NO}_{3}\right)\right]^{+}(\mathbf{3})$ & 416 & 7454 & 188 & 228 & 12994 & 10465 \\
{$\left[(\mathrm{tpm} *)_{2} \mathrm{Ni}\right]^{2+}(\mathbf{4})$} & 423 & 7463 & 500 & 450 & 12923 & 11773 \\
{$\left[(\mathrm{tpm})_{2} \mathrm{Ni}\right]^{2+}(\mathbf{4})$} & 242 & 14358 & 313 & 243 & 12862 & 11765 \\
\hline
\end{tabular}

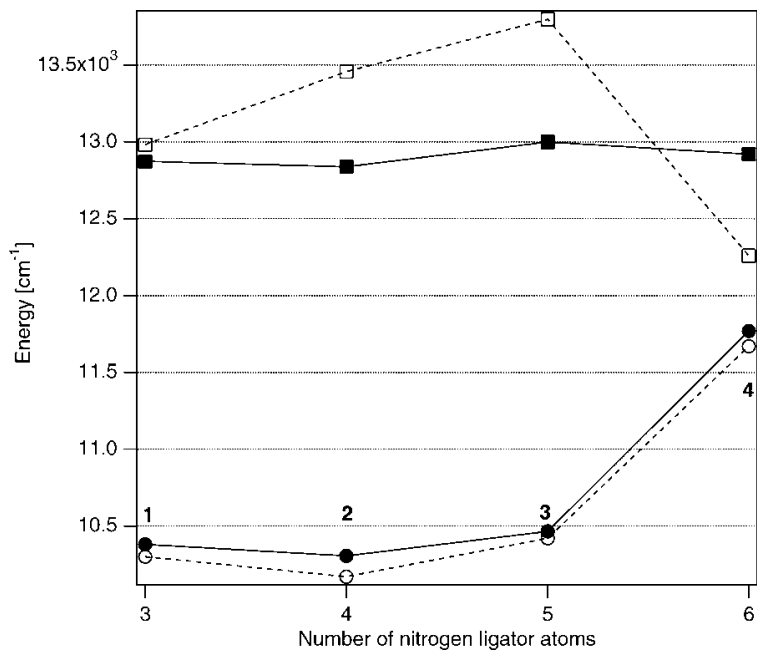

Figure 5. Energies of the lowest-energy singlet (squares, ${ }^{1} \mathrm{E}_{\mathrm{g}}$ in $\mathrm{O}_{\mathrm{h}}$ symmetry) and triplet (circles, ${ }^{3} \mathrm{~T}_{2 \mathrm{~g}}$ in $\mathrm{O}_{\mathrm{h}}$ symmetry) excited states as a function of the number of nitrogen ligator atoms. Solid symbols denote energies obtained from the spectra calculated with (Eq. 5) and shown in Fig. 4. Open symbols denote values obtained from the ligand field parameters in Table 1 with Eqs. 1 and 6.

obtained with different vibrational frequencies cannot be distinguished on the scale of Fig. 4. It is important to note that the energies of the singlet state $\varepsilon_{\mathrm{F}}$ obtained from fits to the absorption spectra using different values for the vibrational frequencies $\omega_{0}$ vary by less than $50 \mathrm{~cm}^{-1}$, illustrating that the model is reliable for the low-symmetry complexes with nonuniform ligand spheres studied here. We have shown and discussed before that this approach avoids the pitfalls of ${ }^{1} \mathrm{E}_{\mathrm{g}}$ energies obtained from phenomenological procedures, such as fitting a sum of Gaussian profiles to the observed absorption bands (14).

\section{Comparison of model parameters}

The model illustrated in Fig. 3 and leading to the calculated spectra in Fig. 4 provides numerical values for the lowest-energy spinallowed and spin-forbidden transitions, summarized in Table 3 as $\Delta$ and $\varepsilon_{\mathrm{F}}$, respectively. They are illustrated for compounds $\mathbf{1}$ to 4 in Fig. 5 and compared to corresponding values from the traditional ligand-field analysis, where the maximum of the lowest-energy spin-allowed band corresponds to 10Dq, as obtained by Eq. 1 and summarized in Table 1 . The comparison of $10 \mathrm{Dq}$ with $\Delta$ shows very similar values and an almost identical variation with the number of nitrogen ligator atoms. The $10 \mathrm{Dq}$ values are systematically lower by approximately $100 \mathrm{~cm}^{-1}$ than those obtained for $\Delta$, a consequence of the omission of spin-orbit coupling in the determination of $10 \mathrm{Dq}$. The magnitude of this difference is on the order expected for spin-orbit coupling effects for complexes of the first-row transition metal ions. Coupling between the singlet and triplet excited states leads to the lower-energy adiabatic potential energy curve, shown as a dotted line in Fig. 3 below the corresponding diabatic curve denoted by a solid line. This diabatic curve defines $\Delta$, and the higher values summarized in Table 3 clearly are a consequence of coupling between the two excited states. The comparison of the maxima for the lowest-energy spinforbidden transition to the ${ }^{1} \mathrm{E}_{\mathrm{g}}$ state is less obvious. The values for $\mathrm{B}$ and 10Dq from Table 1 can be used to calculate the energy of the 
${ }^{1} E_{g}$ excited state. Assuming a constant ratio of the Racah parameters $\mathrm{C} / \mathrm{B}=4$, its energy is given by (13):

$$
\mathrm{E}\left({ }^{1} \mathrm{E}_{\mathrm{g}}\right)=16 \mathrm{~B}-6 \mathrm{~B}^{2} / 10 \mathrm{Dq}
$$

Energies of the ${ }^{1} \mathrm{E}_{\mathrm{g}}$ state calculated with Eq. 7 are compared to the $\varepsilon_{\mathrm{F}}$ values from Table 3 as the top two traces in Fig. 5. The values for $B$ in Table 1 lead to a large variation of $700 \mathrm{~cm}^{-1}$ for the energy of the ${ }^{1} E_{g}$ excited state, in contrast to the energies $\varepsilon_{F}$ obtained from the calculated spectra in Fig. 4, where a variation of less than $160 \mathrm{~cm}^{-1}$ as a function of the number of nitrogen ligator atoms is observed. The parameters from the calculations in Table 3 can be used to obtain alternative values of the ratio $10 \mathrm{Dq} / \mathrm{B}$. The model parameter $\Delta$ corresponds to $10 \mathrm{Dq}$, and Eq. 7 can be used with $\varepsilon_{\mathrm{F}}$ denoting the energy of the ${ }^{1} \mathrm{E}_{\mathrm{g}}$ state to calculate an alternative $\mathrm{B}$ value. The ratio $\Delta / \mathrm{B}$ obtained in this manner is 12.5 for complexes $\mathbf{1}$ to $\mathbf{3}$ and 14.2 for complex $\mathbf{4}$. This set of ratios is closer to the expectations from the spectrochemical and nephelauxetic series than the traditional approach based on the maxima of the spin-allowed transitions and reflects the constant ${ }^{1} \mathrm{E}_{\mathrm{g}}$ energies observed in the absorption spectra of all complexes studied here.

\section{CONCLUSION}

The analysis of readily obtained spectroscopic data such as the solution absorption and solid-state Raman spectra presented here reveals detailed information on fundamental aspects of the electronic structure of complexes with poly(pyrazolyl)methane ligands, an important ligand system forming a variety of low-symmetry transition metal compounds. This new approach leads to a straightforward characterization of the electronic structure and is easily applied to other classes of complexes. Of particular interest could be spectra with several spin-forbidden transitions, providing an opportunity to explore trends obtained from multiple band systems in order to gain more detailed insight into this classic problem in the absorption spectroscopy of transition metal compounds.

Acknowledgements-This work was made possible through research grants from the Natural Sciences and Engineering Research Council (Canada). The authors gratefully acknowledge technical help from N. Baho.

\section{REFERENCES}

1. Kitajima, N. and W. B. Tolman (1995) Coordination chemistry with sterically hindered hydrotris(pyrazolyl)borate ligands: organometallic and bioinorganic perspectives. Prog. Inorg. Chem. 43, 419-527.

2. Zvargulis, E. S., I. E. Buys and T. W. Hambley (1995) Models of the active sites of zinc containing enzymes: the crystal structures of two bis(tripod)zinc(II) complexes. Polyhedron 14, 2267-2273.

3. Kläui, W., B. M., W. Frank, G. J. Reiss, T. Schönherr, G. Rheinwald and H. Lang (2003) Tris(pyrazolyl)methanesulfonates: more than just analogues of tris(pyrazolyl)borate ligands; N,N,N-, N,N,O-, and other coordination modes. Eur. J. Inorg. Chem. 2059-2070.

4. Astley, T., A. J. Canty, M. A. Hitchman, G. L. Rowbottom, B. W. Skelton and A. H. White (1991) Structural, spectroscopic and angularoverlap studies of the nature of metal-ligand bonding for tripod ligands. J. Chem. Soc. Dalton Trans. 1981-1990.

5. Astley, T., J. M. Gulbis, M. A. Hitchman and E. R. T. Tiekink (1993) Structure, spectroscopic and angular-overlap studies of tris(pyrazol1-yl)methane complexes. J. Chem. Soc. Dalton Trans. 509-515.
6. Astley, T., M. A. Hitchman, B. W. Skelton and A. H. White (1997) Structure, electronic spectrum and angular-overlap bonding parameters of bis[bis(pyrazolyl-1.yl)(2-thienyl)-methane](nitrato-O, $\mathrm{O}^{\prime}$ )nickel(II) nitrate. Aust. J. Chem. 50, 145-148.

7. Jørgensen, C. K. (1955) Comparative crystal field studies of some ligands and the lowest singlet state of paramagnetic nickel(II) complexes. Acta Chem. Scand. 9, 1362-1377.

8. Jørgensen, C. K. (1971) Modern Aspects of Ligand-Field Theory. North-Holland, Amsterdam

9. Anthon, C., J. Bendix and C. E. Schäffer (2004) Elucidation of ligandfield theory. reformulation and revival by density functional theory. Struct. Bonding 104, 207-301.

10. Neuhauser, D., T.-J. Park and J. I. Zink (2000) Analytical derivation of interference dips in molecular absorption spectra. Molecular properties and relationship to Fano's antiresonance. Phys. Rev. Lett. 85, 5304-5307.

11. Michaud, A., F.-G. Fontaine and D. Zargarian (2005) Bis[tris(3,5dimethylpyrazolyl)methane]nickel(II). Acta Cryst. E61, m784-m786.

12. Sacconi, L., F. Mani and A. Bencini (1987) Nickel Vol. 5. Comprehensive Coordination Chemistry (Edited by G. Wilkinson, R. Gilard, J. McCleverty), p. 58. Pergamon Press, Oxford, England.

13. Reedijk, J., P. W. N. M. Van Leeuwen and W. L. Groeneveld (1968) A semi-empirical energy-level diagram for octahedral nickel(II) complexes. Recl. Trav. Chim. Pays-Bas 87, 129-143.

14. Nolet, M.-C., R. Beaulac, A.-M. Boulanger and C. Reber (2004) Allowed and forbidden $\mathrm{d}-\mathrm{d}$ bands in octahedral coordination compounds: Intensity borrowing and interference dips in absorption spectra. Struct. Bonding 107, 145-158.

15. König, E. (1971) Simple formulae for crystal-field energies. Struct. Bonding 9, 175-212.

16. Triest, M., G. Bussière, H. Bélisle and C. Reber (2000) Why does the middle band in the absorption spectrum of $\mathrm{Ni}\left(\mathrm{H}_{2} \mathrm{O}\right)_{6}{ }^{2+}$ have two maxima? J. Chem. Educ. 77, 670-670. Available at: http://jchemed.chem.wisc.edu/jcewww/articles/JCENi/JCENi.html. Accessed on 15 September 2005.

17. Schoonhoven, J. W. F. M., W. L. Driessen, J. Reedijk and G. C. Verschoor (1984) Transition-metal co-ordination compounds containing a novel tridentate pyrazole chelating ligand. X-ray crystal structure of [NN-Bis(3,5-dimethylpyrazol-1-ylmethyl)aminoethane](nitratoO)(nitrato-O,O')nickel(II). J. Chem. Soc. Dalton Trans. 1053-1058.

18. Reimann, C. W. (1970) The single crystal spectra of dichlorotetrapyrazolenickel(II), dibromotetrapyrazolenickel(II), and hexapyrazolenickel(II) nitrate. J. Phys. Chem. 74, 561-568.

19. Trofimenko, S. (1993) Recent advances in poly(pyrazolyl)borate (scorpionate) chemistry. Chem. Rev. 93, 943-980.

20. Chanaka, D., L. De Alwis and F. A. Schultz (2003) Metalbis[poly(pyrazolyl)borate] complexes. electrochemical, magnetic, and spectroscopic properties and coupled electron-transfer and spinexchange reactions. Inorg. Chem. 42, 3616-3622.

21. Addison, C. C. and B. M. Gatehouse (1960) The infrared spectra of anhhydrous transition metal nitrates. J. Chem. Soc. 613-615.

22. Rosenthal, M. R. (1973) The myth of the non-coordinating anion. J. Chem. Educ. 50, 331-335.

23. Reedijk, J. and J. Verbiest (1979) Coordination compounds derived from transition metal salts and bis(3,5-dimethylpyrazolyl)methane. Trans. Met. Chem. 4, 239-243.

24. Bussière, G. and C. Reber (1998) Coupled excited states in nickel(II) complexes probed by polarized absorption spectroscopy. J. Am. Chem. Soc. 120, 6306-6315.

25. Bussière, G., C. Reber, D. A. Walter, D. Neuhauser and J. I. Zink (2003) Molecular properties obtained by analysis of electronic spectra containing interference dips. Comparisons of analytical equations and exact models based on coupled potential energy surfaces. J. Phys. Chem. A 107, 1258-1267.

26. Reber, C. and J. I. Zink (1992) Interference dips in molecular absorption spectra calculated for coupled electronic state potential surfaces. J. Chem. Phys. 96, 2681-2690.

27. Fano, U. (1961) Effects of configuration interaction on intensities and phase shifts. Phys. Rev. 124, 1866-1878. 